\title{
Estimulação elétrica funcional associada ao treinamento combinado pós-CRM: ensaio clínico randomizado
}

\author{
Functional electrical stimulation associated with combined post-CABG training: \\ a randomized clinical trial
}

Estimulación eléctrica funcional asociada con el entrenamiento combinado post-CABG: ensayo clinico aleatorizado

Nubia Gonzatti', Letícia Castagna², Maurício Tatsch Ximenes Carvalho ${ }^{3}$, Tamires Daros dos Santos ${ }^{4}$, Dannuey Machado Cardoso ${ }^{5}$, Carine Cristina Callegaro ${ }^{6}$, Isabella Martins de Albuquerque ${ }^{7}$

RESUMO | Os efeitos da adição da estimulação elétrica funcional (EEF) ao treinamento aeróbico e resistido (treino combinado) de curto prazo em pacientes submetidos à cirurgia de revascularização do miocárdio (CRM) ainda não foram estabelecidos. O objetivo do presente estudo é avaliar o impacto da adição da EEF ao treino combinado no fluxo arterial periférico, na capacidade funcional e na qualidade de vida de pacientes pós-CRM participantes de um programa de reabilitação cardíaca - Fase II. Trata-se de um ensaio clínico randomizado, duplo cego, composto por 17 pacientes (54,8 $\pm 10,5$ anos, 12 homens) randomizados ou em grupo intervenção (Gl, $n=8$,) submetido à EEF no músculo quadríceps associada ao treino combinado, ou em grupo sham (GS, n=9), que realizou a EEF sham em associação ao treino combinado. Os desfechos avaliados foram: fluxo arterial periférico (índice tornozelo-braquial), capacidade funcional (distância percorrida no teste de caminhada de seis minutos - DTC6M) e qualidade de vida (questionário MacNew). Na comparação entre os grupos, o aumento do índice tornozelo-braquial

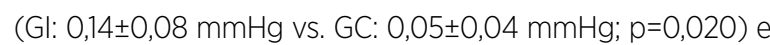
do escore do domínio global do questionário MacNew (Gl: 1,1 $\pm 0,3$ pontos vs. GC: 0,6 $\pm 0,4$ pontos; $p=0,020$ ) foi maior no Gl. Entretanto, não foi observada diferença entre os grupos

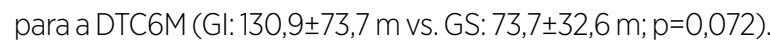
A adição da EEF, durante curto período, potencializou os efeitos do exercício aeróbico e resistido sobre o fluxo arterial periférico e a qualidade de vida em pacientes pós CRM em Fase II da reabilitação cardíaca.

Descritores | Estimulação Elétrica; Reabilitação Cardíaca; Revascularização Miocárdica; Índice Tornozelo-Braço; Qualidade de Vida.

\begin{abstract}
The effects of adding functional electrica stimulation (FES) to short-term aerobic and resistance exercise (combined training) in patients undergoing coronary artery bypass graft (CABG) surgery have not yet been established. This study aims to evaluate the effect of adding FES to combined training on peripheral arterial flow, functional capacity and
\end{abstract}

\footnotetext{
'Universidade Federal de Santa Maria (UFSM) - Santa Maria (RS), Brasil. E-mail: nubia_gonzatti@hotmail.com. Orcid: 0000-0001-8570-598X

2Universidade Federal de Santa Maria (UFSM) - Santa Maria (RS), Brasil. E-mail: leticiacastagnaa@gmail.com. Orcid: 0000-0002-1466-7872

${ }^{3}$ Centro Universitário da Região da Campanha (URCAMP) - Bagé (RS), Brasil. E-mail: mauximen96@hotmail.com. Orcid: 0000-0001-6679-2854

${ }^{4}$ Universidade Federal de Santa Maria (UFSM) - Santa Maria (RS), Brasil. E-mail: tamires.daros@gmail.com. Orcid: 0000-0002-4637-4185

5Universidade Federal do Rio Grande do Sul (UFRGS) - Porto Alegre (RS), Brasil. E-mail: dannuey@yahoo.com.br. Orcid: 0000-0003-2891-1028

6Universidade Federal de Santa Maria (UFSM) - Santa Maria (RS), Brasil. E-mail: ccallegaro84@gmail.com. Orcid: 0000-0001-9482-5457

7 Universidade Federal de Santa Maria (UFSM) - Santa Maria (RS), Brasil. E-mail: albuisa@gmail.com.

Orcid: 0000-0002-7256-1881
} 
quality of life of post-CABG patients participating in a cardiac rehabilitation program - Phase II. This is a randomized, double-blind, clinical trial, composed of 17 patients ( $54.8 \pm 10.5$ years old, 12 men) randomized or in an intervention group ( $\mathrm{IG}, \mathrm{n}=8$,) submitted to FES in the quadriceps muscle associated with combined training, or in a sham group ( $\mathrm{SG}, \mathrm{n}=9$ ), which performed the FES sham in association with the combined training. The evaluated outcomes were: peripheral arterial flow (ankle-brachial index), functional capacity (distance covered in the six-minute walk test - 6MWT) and quality of life (MacNew questionnaire). In the comparison between the groups, the increase in the ankle-brachial index (IG: $0.14 \pm 0.08 \mathrm{mmHg}$ vs. CG: $0.05 \pm 0.04 \mathrm{mmHg} ; \mathrm{p}=0.020$ ) and the score of the global MacNew questionnaire (IG: $1.1 \pm 0.3$ points vs. CG: $0.6 \pm 0.4$ points; $p=0.020$ ) was higher in the IG. However, no difference was observed between the

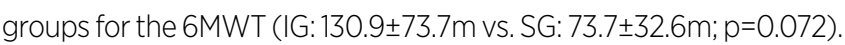
The addition of FES, during a short period, potentiated the effects of aerobic and resistance exercise on peripheral arterial flow and quality of life in patients after CABG in Phase II of cardiac rehabilitation. Keywords | Electrical stimulation; Cardiac Rehabilitation; Coronary Artery Bypass; Ankle-brachial Index; Quality of life.

RESUMEN I Aún no se han establecido los efectos de agregar estimulación eléctrica funcional (EPS) cortoplacista al entrenamiento aeróbico y de resistencia (entrenamiento combinado) en pacientes sometidos a cirugía de injerto de derivación de las arterias coronarias (CABG). El objetivo del presente estudio fue evaluar el impacto de la adición de EPS al entrenamiento combinado sobre el flujo arterial periférico, la capacidad funcional y la calidad de vida de los pacientes post-CABG que participan en un programa de rehabilitación cardíaca de Fase II. Este es un ensayo clínico aleatorizado, doble ciego, compuesto por 17 pacientes ( $54,8 \pm 10,5$ años, 12 hombres) aleatorizados en un grupo de intervención ( $G$ l, $n=8$ ) sometidos a EEF en el músculo cuádriceps asociado a entrenamiento combinado o en grupo simulado (GS, n=9), que realizó el simulacro de EEF en asociación con el entrenamiento combinado. Los resultados evaluados fueron: flujo arterial periférico (índice tobillo braquial), capacidad funcional (distancia recorrida en la prueba de caminata de seis minutos - 6MWT) y calidad de vida (cuestionario MacNew). Al comparar los grupos, el aumento del

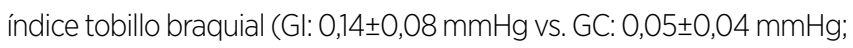
$p=0,020)$ y la puntuación para el dominio global del cuestionario

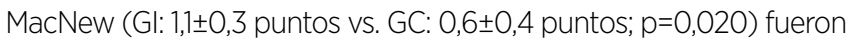
mayores en el Gl. Sin embargo, no hubo diferencias entre los grupos

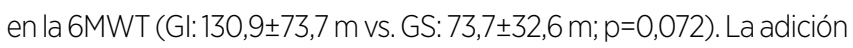
de EEF, durante un período corto, potenció los efectos del ejercicio aeróbico y de resistencia sobre el flujo arterial periférico y la calidad de vida en pacientes post-CABG en la Fase II de rehabilitación cardíaca. Palabras clave | Estimulación Eléctrica; Rehabilitación Cardiaca; Revascularización Miocárdica; Índice Tobillo Braquial; Calidad de Vida.

\section{INTRODUÇÃO}

Doenças cardiovasculares compreendem as principais causas de morte e de invalidez no Brasil e no mundo, sendo a doença arterial coronariana (DAC) responsável por 7,4 milhões dessas mortes em nível global ${ }^{1}$. O tratamento da DAC pode ser clínico ou cirúrgico, dependendo dos benefícios da terapêutica frente a condição clínica do paciente ${ }^{2}$. A cirurgia de revascularização do miocárdio (CRM) é eficaz na redução dos sintomas e mortalidade em pacientes com $\mathrm{DAC}^{2}$, porém apresenta implicações clínicas e funcionais, que podem inclusive acometer o sistema muscular esquelético ${ }^{3}$.

Conforme estudos prévios, o estresse cirúrgico ocasiona desregulação do metabolismo proteico decorrente do hipercatabolismo pós-operatório, o que consequentemente induz a perda de massa muscular e a redução persistente da força muscular ${ }^{4,5}$. Tais alterações têm sido atribuídas à reação inflamatória sistêmica ${ }^{4}$. Ademais, o comprometimento muscular periférico contribui para o declínio funcional e para a redução da qualidade de vida em pacientes submetidos à cirurgia cardíaca $(\mathrm{CC})^{3,4}$.

Neste sentido, evidências preconizam a instituição da reabilitação cardíaca $(\mathrm{RC})$, cuja estratégia típica compreende exercícios aeróbicos e resistidos (treino combinado TC). No entanto, alguns pacientes são intolerantes aos exercícios de baixa intensidade ${ }^{6,7}$. Adicionalmente, frente às alterações no sistema muscular esquelético após $\mathrm{CC}$, é notório o interesse quanto ao uso de recursos terapêuticos que possam minimizar tais efeitos, como a estimulação elétrica funcional $(\mathrm{EEF})^{8}$.

A EEF compreende a aplicação de estímulos intermitentes e superficiais em músculos esqueléticos, visando ativar ramos nervosos e, assim, estimular contrações visíveis ${ }^{9}$. Revisões sistemáticas têm evidenciado que o uso da EEF é capaz de promover aumento da força e resistência do quadríceps e da capacidade funcional (CF) em pacientes com doença pulmonar obstrutiva crônica e melhorar a capacidade de exercício, a qualidade de vida, a força muscular, a função endotelial e os sintomas depressivos em pacientes com insuficiência cardíaca (IC) ${ }^{10,11}$. Entretanto, a literatura é ainda escassa sobre o potencial efeito da EEF associada à $\mathrm{RC}$ em pacientes pós CRM. 
Dentro desse contexto, o objetivo do estudo foi avaliar o impacto da adição da EEF ao exercício aeróbico e de resistência (TC) no fluxo arterial periférico, CF e qualidade de vida de pacientes pós CRM participantes de um programa de $\mathrm{RC}-$ Fase II.

\section{MÉTODOS}

\section{Delineamento do estudo}

Ensaio clínico randomizado duplo-cego, registrado no ClinicalTrials.gov (Identifier: NCT03560713), realizado no Programa de Reabilitação Cardíaca da Unidade de Reabilitação do Hospital Universitário de Santa Maria (HUSM) da Universidade Federal de Santa Maria (UFSM), em Santa Maria (RS), Brasil, no período de abril de 2017 a junho de 2018. Todos os participantes assinaram o termo de consentimento livre e esclarecido antes da inclusão no estudo, conforme determinação da resolução no 466/12 do Conselho Nacional de Saúde.

\section{PARTICIPANTES}

Os critérios de elegibilidade incluíram pacientes submetidos à CRM, com idade entre 50 e 70 anos, clinicamente estáveis, recrutados da lista de espera para a Fase II da RC do hospital supracitado e com anuência da equipe médica para prática de exercício físico. Foram excluídos sujeitos com instabilidade clínica e hemodinâmica, problemas ortopédicos, neurológicos graves, alterações vasculares periféricas nos membros inferiores (trombose venosa profunda, tromboangeite obliterante e doença arterial obstrutiva-DAOP), doença pulmonar obstrutiva crônica, doença cerebrovascular ou que apresentassem as seguintes contraindicações ao uso da EEF: lesões epidérmicas no local da aplicação, intolerância à estimulação elétrica, alteração na sensibilidade da pele ou uso de marcapasso.

\section{Randomização}

Os sujeitos incluídos no estudo foram randomizados em dois grupos: grupo intervenção (GI), que realizou a EEF adicionalmente ao treinamento combinado (exercício aeróbico e de resistência), e grupo sham (GS), submetido à $\mathrm{EEF}$ sham em associação com o TC. A randomização em blocos foi realizada por um colaborador externo ao estudo, através de uma ordem aleatória de alocação, utilizando os números 1 e 2 para designar os sujeitos do GI e do GS respectivamente, através do software Random Number Generator (Pro v2.00, Segobit, Issaquah, WA, EUA).

Visando garantir o duplo cegamento, os pacientes não foram informados sobre o grupo de alocação e a EEF foi realizada por uma pesquisadora que não participou da avaliação das medidas de desfechos.

\section{Procedimentos}

Os pacientes foram submetidos a dois dias de avaliações, com intervalo mínimo de 48 horas entre eles. No primeiro dia, foram realizadas a anamnese, o exame físico e a avaliação da CF. No segundo dia foi realizada a aplicação do questionário MacNew e a avaliação do índice tornozelobraquial (ITB). Todas as avaliações foram realizadas por avaliadores previamente treinados.

\section{Avaliação da capacidade funcional (CF)}

A CF foi avaliada através do teste de caminhada de seis minutos (TC6M) conforme recomendações preconizadas pela American Thoracic Society (ATS) ${ }^{12}$. A distância percorrida foi mensurada em metros e os valores obtidos foram comparados com o previsto conforme as equações de Enright e Sherrill ${ }^{13}$.

\section{Avaliação da qualidade de vida}

A versão em português, traduzida e adaptada para sujeitos cardiopatas, do questionário MacNew Heart Disease Healthrelated Quality of Life Questionnaire (MacNew QLMI) foi utilizada para a avaliação da qualidade de vida sob a forma de entrevista. Para o cálculo da pontuação obtida no questionário, foram utilizadas as médias das pontuações dos domínios físico, social e emocional. Uma maior pontuação obtida no MacNew corresponde a uma melhor qualidade de vida ${ }^{14}$.

\section{Avaliação do fluxo arterial periférico}

O fluxo arterial periférico foi avaliado através da medida do ITB de membros inferiores pelo método da ultrassonografia Doppler (USG Doppler) de alta resolução (EnVisor HD, Philips, Eindhoven, Holanda) com transdutor linear multifrequencial de 7 a $12 \mathrm{MHz}$. O paciente foi posicionado em decúbito dorsal e as artérias braquiais, pediosas e tibiais posteriores foram incluídas na avaliação do $\mathrm{ITB}^{15}$. O protocolo de avaliação foi realizado através de um esfigmomanômetro aneróide (G-TECH/Premium) posicionado logo acima do 
maléolo e insuflado até a perda da pulsação arterial, avaliada com auxílio de um estetoscópio (Littmann Classic III 5803). Após o transdutor da ultrassonografia, foi colocado sobre a artéria e o esfigmomanômetro desinsuflado. A pressão arterial sistólica (PAS) de membros superiores (MMSS) também foi avaliada.

O cálculo utilizado para o ITB compreendeu a divisão do valor da PAS mais alta dos músculos dos MMII pelo valor mais elevado da PAS nos músculos dos MMSS ${ }^{15}$. A classificação do ITB teve como base os seguintes valores: 1,00 a 1,40 $\mathrm{mmHg}$ (normal), 0,91 a 0,99 $\mathrm{mmHg}$ (limítrofe) e indicativo de DAOP menor que $0,90^{16}$.

\section{Protocolo de Reabilitação Cardíaca (Fase II)}

Todos os pacientes foram inseridos no programa de $\mathrm{RC}$ (Fase II), cujo treinamento físico foi composto por exercício aeróbico e de resistência, realizado duas vezes por semana durante 12 semanas, de acordo com protocolo baseado em estudo prévio ${ }^{17}$. Os exercícios aeróbicos foram realizados em esteira ergométrica (Inbramed, ATL-10200, RS, BR), durante 30 minutos, com a intensidade de treinamento baseada na frequência cardíaca (FC) obtida durante o teste ergométrico. A FC de treinamento foi calculada através da diferença entre $\mathrm{FC}$ máxima e $\mathrm{FC}$ de repouso, com intensidade de 55-65\% e uma pontuação de 4-6 na escala de Borg modificada (variando de 0 a 10) ${ }^{17}$.

Os exercícios de resistência para membros superiores e inferiores foram realizados com halteres e caneleiras por aproximadamente 20 minutos, sendo executadas 3 séries de 10 repetições por grupo muscular, com intervalo de repouso de 30 segundos. O exercício resistido iniciou com $40 \%$ do teste de repetição máxima e prosseguiu até o paciente atingir $60 \%$, 8 . De modo complementar, foram realizados exercícios com faixa elástica (Thera-band ${ }^{\circledR}$ ), cuja resistência foi estimada com base na cor que apresentavam. No início e ao término de cada sessão foram realizados 10 minutos de alongamento. A monitorização dos sinais vitais foi realizada de forma constante.

\section{Protocolo de estimulação elétrica funcional (EEF)}

No primeiro dia de intervenção foram identificados, a partir da varredura da área, os pontos motores do quadríceps femoral através de um eletrodo superior posicionado $4 \mathrm{~cm}$ abaixo da dobra inguinal, enquanto o eletrodo inferior foi colocado acima da patela em ambos os membros inferiores ${ }^{18}$. A EEF foi realizada com o paciente em decúbito dorsal, mantendo flexão do joelho de $60^{\circ}$. A aplicação foi efetuada através de um estimulador transcutâneo neuromuscular calibrado (Neurodyn High Volt, IBRAMED, Amparo, SP, Brasil) por meio de eletrodos autoadesivos retangulares de 5 por $9 \mathrm{~cm}$, nos quais foram posicionados nos pontos motores, com frequência de $25 \mathrm{~Hz}$, duração de pulso de $200 \mu$ s, tempo de contração de 5 segundos, tempo de repouso de 5 segundos, rampa de subida e descida de 1 segundo e intensidade da corrente a mais alta possível a ser tolerada por paciente do $\mathrm{GI}^{18}$.Já no GS, a frequência utilizada foi de $5 \mathrm{~Hz}$, o que visava não provocar uma contração muscular visível ${ }^{18}$. A intervenção foi realizada com uma frequência de três sessões semanais durante 12semanas (duração de 30minutos cada sessão), sendo que duas sessões semanais eram realizadas antes do programa de $\mathrm{RC}$ e, na outra sessão, o paciente realizava conforme agendamento no ambulatório. A monitorização dos sinais vitais foi realizada de forma constante.

\section{Cálculo do tamanho amostral}

A amostra deste estudo piloto foi utilizada para inferência amostral do ensaio clínico randomizado, estimado para obter um nível de significância de $5 \%(\mathrm{p}<0,05)$ e poder de 80\% (GPower 3.1), considerando-se uma diferença entre as médias do ITB de 0,008 $\pm 0,11 \mathrm{mmHg}$ e um tamanho amostral necessário de 9 pacientes em cada grupo.

\section{Análise estatística}

Os dados foram analisados no software Statistical Package for the Social Sciences, versão 20.0 (SPSS Inc., Chicago, IL, EUA). A normalidade das variáveis foi avaliada pelo teste de Shapiro-Wilk. As variáveis contínuas foram apresentadas na forma de média \pm desvio-padrão e intervalo de confiança de 95\% (IC 95\%), enquanto as categóricas foram apresentadas em frequências absolutas e porcentagens. Para comparar os momentos pré e pósintervenção intragrupo, foi utilizado o teste t Student pareado. A comparação entre os grupos realizou-se através do teste $\mathrm{t}$ Student não pareado. Para efeito de significância estatística, foi considerado um $\mathrm{p}<0,05$.

\section{RESULTADOS}

O diagrama de fluxo apresenta os pacientes potencialmente elegíveis e os de fato incluídos no presente estudo (GS=9, $\mathrm{GI}=8$ ) (Figura 1). Durante o estudo, não foram observados eventos adversos em relação ao protocolo. Todos os pacientes completaram as 36 sessões de EEF ao longo das 12semanas. 


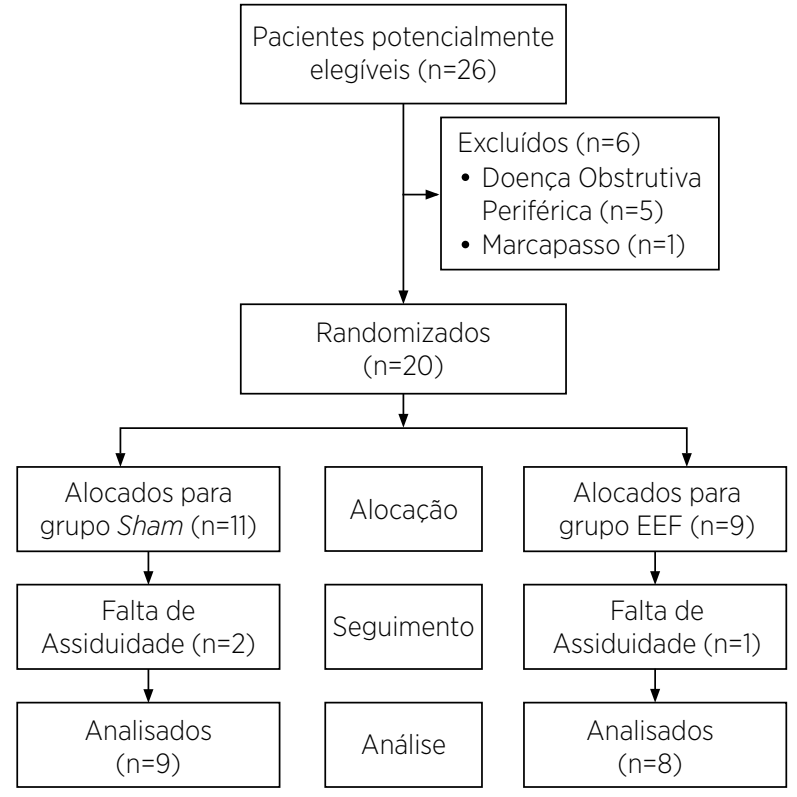

Figura 1. Fluxograma do estudo

$\mathrm{Na}$ Tabela 1 são apresentadas as características clínicas e demográficas dos pacientes inseridos no estudo.

Tabela 1. Características clínicas e demográficas dos pacientes

\begin{tabular}{lrr}
\multicolumn{1}{c}{ Variáveis } & GI $(\mathrm{n}=8)$ & GS $(\mathrm{n}=9)$ \\
Idade, anos & $51,6 \pm 12,4$ & $57,7 \pm 7,2$ \\
Sexo masculino, $\mathrm{n}(\%)$ & $5(62,5)$ & $5(55,6)$ \\
IMC, Kg/m² & $28,8 \pm 2,2$ & $28,0 \pm 2,5$ \\
FEVE, \% & $59,1 \pm 14,9$ & $60,4 \pm 12,8$ \\
DM, n (\%) & $5(62,5)$ & $5(55,6)$ \\
HAS, n (\%) & $6(75,0)$ & $8(88,9)$ \\
Início da RC pós CRM, dias & $35,8 \pm 2,1$ & $38,4 \pm 1,7$ \\
Medicamentos, n (\%) & & \\
AINEs & $5(62,5)$ & $9(100,0)$ \\
Antiplaquetário & $2(25,0)$ & \\
Estatina & $6(75,0)$ & $9(100,0)$ \\
Diurético & $3(37,5)$ & $5(55,6)$ \\
Anticoagulante & - & $1(11,1)$ \\
IECA & $6(75,0)$ & $1(11,1)$ \\
Beta-bloqueador & $5(62,5)$ & $7(77,8)$
\end{tabular}

Valores expressos em média e desvio-padrão; GS :grupo sham (EEF sham + treino combinado); Gl: grupo intervenção (EEF + treino combinado); IMC: índice de massa corporal; FEVE: fração de ejeção do ventrículo esquerdo; DM: diabetes mellitus; HAS: hipertensão arterial sistêmica; RC: reabilitação cardíaca; CRM: cirurgia de revascularização do miocárdio; AINEs: anti-inflamatórios não esteroides; IECA: inibidores da enzima conversora de angiotensina.
A Figura 2 demonstra o aumento do ITB observado na comparação intragrupo tanto no GI (pré: $1,05 \pm 0,05$ $\mathrm{mmHg}$; pós: $1,19 \pm 0,08 \mathrm{mmHg} ; \mathrm{p}=0,002)$ quanto no $\mathrm{GS}$

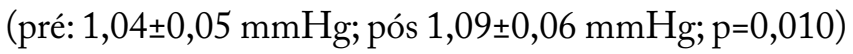
após a implementação do protocolo. $\mathrm{Na}$ comparação entre as diferenças apresentadas pelos grupos, nos momentos pré e pós protocolo, foi observado incremento significativo no ITB em favor do GI $(0,14 \pm 0,08 \mathrm{mmHg}$ vs. 0,05 $\pm 0,04 \mathrm{mmHg} ; \mathrm{p}=0,020$ ).

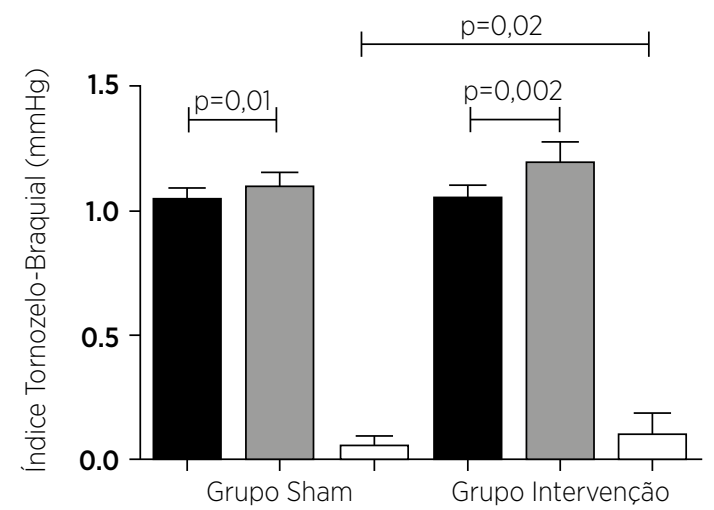

Pré-intervenção $\square$ Pós-intervenção $\square$ Diferença

Figura 2. Comparação intra e entre grupos do fluxo arterial periférico avaliado por meio do índice tornozelo-braquial nos momentos avaliados

$\mathrm{Na}$ comparação intragrupo, houve aumento significativo na DTC6M no GI $(130,9 \pm 73,7 \mathrm{~m}, \mathrm{IC}$ $95 \% 69,3$ a 192,5$)$ e no GS $(73,7 \pm 32,6 \mathrm{~m}, \mathrm{IC} 95 \%$ $48,7$ a 98,8$)$, porém não foi observada diferença significativa entre os grupos $(57,2 \pm 27,1 \mathrm{~m}, \mathrm{IC} 95 \%-0,5$ a 114,7). Considerando o escore do domínio global do questionário MacNew, observou-se aumento significativo na comparação intragrupo tanto no $\mathrm{GI}(1,1 \pm 0,3$ pontos, IC $95 \% 0,8$ a 1,3$)$ quanto no GS $(0,6 \pm 0,4$ pontos, IC $95 \% 0,2$ a 0,9$)$ (Tabela 2). Na comparação entre os grupos, foi observado incremento significativo na pontuação obtida no questionário $\mathrm{MacNew}$ em favor do GI após o protocolo $(0,5 \pm 0,2$ pontos, IC $95 \%$ $0,09$ a 0,9$)$ (Tabela 2).

Tabela 2. Capacidade funcional submáxima e qualidade de vida.

\begin{tabular}{|c|c|c|c|c|c|c|c|c|}
\hline \multirow[b]{2}{*}{ Variáveis } & \multicolumn{3}{|c|}{$\mathrm{GI}(n=8)$} & \multicolumn{3}{|c|}{$\mathrm{GS}(n=9)$} & \multicolumn{2}{|c|}{$\begin{array}{l}\text { Comparação } \\
\text { entre grupos }\end{array}$} \\
\hline & Pré & Pós & $p$ & Pré & Pós & $p$ & $\Delta$ entre grupos & $p$ \\
\hline TC6M & $414,7 \pm 49,8$ & $545,6 \pm 60,8$ & $0,002^{* a}$ & $414,7 \pm 44,9$ & $488,4 \pm 41,9$ & $<0,001^{* a}$ & $57,2 \pm 27,1$ & $0,072^{a}$ \\
\hline QV & $4,5 \pm 0,6$ & $5,6 \pm 0,5$ & $<0,001^{* a}$ & $4,5 \pm 0,7$ & $5,1 \pm 0,6$ & $0,004^{* a}$ & $0,5 \pm 0,2$ & $0,020^{\star a}$ \\
\hline
\end{tabular}

Valores expressos em média e desvio-padrão. Gl: grupo intervenção (EEF + treino combinado); GS: grupo sham (EEF sham + treino combinado; TC6M: teste de caminhada de seis minutos; QV: qualidade de vida (escore do domínio global do questionário MacNew). $\Delta$ : variação: " significância estatística p<0,05. äTeste T Student. 


\section{DISCUSSÃO}

Neste ensaio clínico randomizado, investigamos os efeitos da adição da EEF ao TC (exercício aeróbico e de resistência) em pacientes pós CRM inseridos na Fase II da RC. Nossos achados demonstraram que a adição do referido recurso terapêutico foi capaz de potencializar os efeitos do TC sobre o aumento do fluxo arterial periférico e a melhora da qualidade de vida. Entretanto, a EEF não propiciou benefícios adicionais na $\mathrm{CF}$.

Nossos resultados estão em conformidade com estudos prévios, os quais demonstraram que a implementação da EEF no quadríceps femoral promoveu aumento da função endotelial, mensurada através da dilatação mediada pelo fluxo da artéria braquial, de pacientes com $\mathrm{IC}^{18,19}$. Dessa forma, sugere-se que as alterações benéficas induzidas pela EEF na hemodinâmica periférica e no estado metabólico muscular, bem como a reversão do descondicionamento físico poderiam explicar a melhora da função endotelia ${ }^{20}$. No entanto,é importante mencionar que nosso estudo difere dos citados anteriormente pelo fato de recrutarmos pacientes pós CRM; além disso, a mensuração do fluxo arterial periférico foi realizada por meio do ITB, que tem sido considerado um método simples, reprodutível, não invasivo e de baixo custo $^{21}$. Ressalta-se que a redução no ITB é associada com a piora da função cardíaca, a gravidade da DAC, o aumento do risco de revascularização da lesão alvo e de eventos cardíacos e cerebrovasculares adversos importantes ${ }^{22}$.

Adicionalmente, torna-se relevante destacar que mesmo utilizando um protocolo de EEF de curto prazo, com frequência de 3 vezes por semana em associação ao TC, foi constatado um aumento do fluxo arterial periférico. Sabe-se que a EEF induz atividade muscular com aumento da captação de oxigênio para o músculo, culminando em redistribuição do fluxo sanguíneo, melhora da perfusão capilar e aumento do fluxo sanguíneo intramuscular ${ }^{23}$. O treinamento aeróbico também promove aumento do fluxo arterial periférico, decorrente do aumento no débito cardíaco e da capacidade de perfusão muscular, permitindo maior liberação de oxigênio e, assim, minimizando a resistência ao fluxo $^{24}$. De modo semelhante, o treinamento de resistência pode induzir a adaptações hemodinâmicas e estruturais, como aumento na capacidade de vasodilatação do endotélio e da extração de oxigênio pelo músculo, que influenciam o sistema musculoesquelético ${ }^{20,24}$. Diante do exposto, a possível explicação para o aumento do fluxo arterial periférico constatado em nosso estudo pode estar relacionada ao fato de que a EEF foi associada ao TC. Em contraste com achados demonstrados em estudos prévios ${ }^{17,19}$, nosso estudo demonstrou que, após um período de 12 semanas de EEF associada ao TC, houve aumento do fluxo arterial periférico em pacientes pós CRM. Essa divergência pode ser explicada pela duração do protocolo de apenas 6 semanas de EEF sem associação a treinamento em indivíduos com $\mathrm{ICC}^{17,19}$.

A CRM propicia melhora clinicamente importante na qualidade de vida no pós-operatório tardio. Premissa essa evidenciada por estudo prospectivo, ao demonstrar que um mês após a cirurgia esse desfecho ainda era inadequado. No entanto, resultados satisfatórios foram relatados um ano após o procedimento cirúrgico ${ }^{25}$. Neste sentido, investigar recursos terapêuticos que possam melhorar a qualidade de vida a curto prazo torna-se relevante clinicamente. Conforme nossos achados, a EEF pode ser um desses recursos, pois foi capaz de potencializar os efeitos do TC na melhora da qualidade de vida no grupo intervenção em comparação ao grupo-controle após, aproximadamente, 3 meses da CRM. Tal resultado está em conformidade aos achados de um estudo piloto conduzido por Parissis et al. ${ }^{19} \mathrm{em} 30$ pacientes idosos com IC crônica ao demonstrarem que a EEF, com frequência de $25 \mathrm{~Hz}$, tempo de contração de $5 \mathrm{~s}$, tempo de repouso de $5 \mathrm{~s}$, intensidade ajustada no limite da tolerância do paciente, por 30 minutos, 5 vezes por semana, durante 6 semanas nos músculos quadríceps e gastrocnêmio, foi capaz de promover melhora da qualidade de vida.

A EEF não foi capaz de propiciar benefícios adicionais quando associada ao TC no desfecho relativo à capacidade de exercício. Achado esse corroborado por ensaio clínico randomizado conduzido em 20 pacientes pós $\mathrm{CC}$, no qual a EEF, com frequência de $15 \mathrm{~Hz}$, tempo de contração de $5 \mathrm{~s}$, tempo de repouso de 10s, intensidade no limite da tolerância do paciente, por 40 minutos, 2 vezes por semana, durante 8 semanas no músculo quadríceps, ocasionou aumento na DTC6M intragrupo (grupo FES e grupo FES placebo), porém sem haver diferença significativa entre os grupos?. Em contraste, Karavidas et al. ${ }^{18}$ evidenciaram incremento na $\mathrm{CF}$ ao investigar os efeitos da $\mathrm{EEF}$, com frequência de $25 \mathrm{~Hz}$, tempo de contração de $5 \mathrm{~s}$, tempo de repouso de $5 \mathrm{~s}$, intensidade tolerada pelo paciente, por 30 minutos, 5 vezes por semana, durante 6 semanas nos músculos quadríceps e gastrocnêmio, em 30 pacientes com ICC e fração de ejeção ventricular esquerda preservada. Infere-se que tal achado divergente pode estar relacionado ao fato de que no estudo anteriormente citado o uso da EEF foi comparado com o placebo; já no presente estudo, comparamos o referido recurso terapêutico com uma co-intervenção. Ressalta-se que o protocolo de EEF utilizado em nosso estudo foi similar ao de Karavidas et al. ${ }^{18}$, com exceção dos grupos musculares. Tornase relevante considerar que a eficácia da EEF é dependente 
das propriedades neuromusculares intrínsecas individuais, que são superiores aos fatores externamente controláveis (parâmetros da EEF) na determinação do nível de tensão gerada no músculo ${ }^{9}$. Cabe destacar que, apesar dos pacientes receberem orientações para não realizarem outra forma de exercício em sua vida cotidiana durante a intervenção proposta, não foi possível o controle de tal variável, o que poderia ter ocasionado implicações no desfecho da CF.

Este estudo apresenta algumas limitações que devem ser consideradas. Em primeiro lugar, a ausência de um grupocontrole composto por pacientes submetidos à CRM, mas que não tivessem disponibilidade de realizar as intervenções propostas, visando avaliar o processo de recuperação natural da doença após o procedimento cirúrgico. Além disso, não foi possível realizar uma análise por intenção de tratar. Estudos futuros com maior tamanho amostral e maior tempo de seguimento poderiam reforçar nossos achados, principalmente em relação ao desfecho da CF.

Embora ocorram limitações, nossos achados apresentam relevância para a prática clínica, pois propiciam uma nova perspectiva quanto ao uso da EEF como complemento ao treinamento físico, sendo um recurso de baixo custo e fácil inserção em programas de RC Fase II.

\section{CONCLUSÃO}

Este ensaio clínico randomizado demonstrou que a adição da EEF, mesmo que conduzida por um período relativamente curto e com frequência semanal reduzida, foi capaz de potencializar os efeitos do TC sobre o fluxo arterial periférico e a qualidade de vida em pacientes pós CRM inseridos na Fase II da RC. Nossos achados também ratificam o uso da EEF como uma intervenção terapêutica complementar ao treinamento físico, sendo um recurso seguro, de baixo custo e com boa adesão.

\section{REFERÊNCIAS}

1. Organização Pan-Americana de Saúde. Doenças cardiovasculares [Internet]. Brasília; 2017 [cited 2020 July 29]. Available from: https://www.paho.org/bra/index.php?option=com_content\&v iew=article\&id=5253:doencas-cardiovasculares\&ltemid=1096

2. Kandaswamy E, Zuo L. Recent advances in treatment of coronary artery disease: role of science and technology. Int J Mol Sci. 2018;19(2):424. doi: 10.3390/ijms19020424

3. Van Venrooij LM, Verberne HJ, de Vos R, Borgmeijer-Hoelen MM, van Leeuwen PA, de Mol BA. Postoperative loss of skeletal muscle mass, complications and quality of life in patients undergoing cardiac surgery. Nutrition. 2012;28:40-5. doi: 10.1016/j.nut.2011.02.007
4. lida Y, Yamazaki T, Arima H, Kawabe T, Yamada S. Predictors of surgery-induced muscle proteolysis in patients undergoing cardiac surgery. J Cardiol. 2016;68(6):536-41. doi: 10.1016/j. jjcc.2015.11.011

5. Santos KM, Cerqueira Neto ML, Carvalho VO, Santana Filho VJ, Silva Junior WM, Araújo Filho AA, et al. Evaluation of peripheral muscle strength of patients undergoing elective cardiac surgery: a longitudinal study. Rev Bras Cir Cardiovasc. 2014;29(3):355-9. doi: 10.5935/1678-9741.20140043

6. Herdy AH, López-Jimenez F, Terzic CP, Milani M, Stein R, Carvalho $\mathrm{T}$, et al. South American guidelines for cardiovascular disease prevention and rehabilitation. Arq Bras Cardiol. 2014;103(2 Suppl 1):1-31. doi: 10.5935/abc.2014s003

7. Smart NA, Dieberg G, Giallauria F. Functional electrical stimulation for chronic heart failure: a meta-analysis. Int J Cardiol. 2013;167(1):80-6. doi: 10.1016/j.ijcard.2011.12.019

8. Schardong J, Kuinchtner GC, Sbruzzi G, Plentz RDM, Silva AMVD. Functional electrical stimulation improves muscle strength and endurance in patients after cardiac surgery: a randomized controlled trial. Braz J Phys Ther. 2017;21(4):26873. doi: 10.1016/j.bjpt.2017.05.004

9. Maffiuletti NA, Gondin J, Place N, Stevens-Lapsley J, Vivodtzev I, Minetto MA. Clinical use of neuromuscular electrical stimulation for neuromuscular rehabilitation: what are we overlooking? Arch Phys Med Rehabil. 2018;99:806-12. doi: 10.1016/j. apmr.2017.10.028

10. Hill K, Cavalheri V, Mathur S, Roig M, Janaudis-Ferreira T, Robles P, Dolmage TE, Goldstein R. Neuromuscular electrostimulation for adults with chronic obstructive pulmonary disease (Review). Cochrane Database Syst Rev. 2018;29(5):1-76. doi: 10.1007/ BF00581062

11. Gomes Neto M, Oliveira FA, Reis HFC, Rodrigues ES, Bittencourt HS, Carvalho VO. Effects of neuromuscular electrical stimulation on physiologic and functional measurements in patients with heart failure: a systematic review with meta-analysis. J Cardiopulm Rehabil Prev. 2016;36(3):157-66. doi: 10.1097/ HCR.0000000000000151

12. American Thoracic Society. ATS Statement: Guidelines for the six-minute walk test. Am J Respir Crit Care Med. 2002;166(1):1117. doi: 10.1164/ajrccm.166.1.at1102

13. Enright PL, Sherrill DL. Reference equations for the sixminute walk in healthy adults. Am J Respir Crit Care Med. 1998;158(5):1384-7. doi: 10.1164/ajrccm.158.5.9710086

14. Nakajima KM, Rodrigues RC, Gallani MC, Alexandre NM, Oldridge N. Psychometric properties of MacNew Heart Disease Healthrelated Quality of Life Questionnaire: Brazilian version. J Adv Nurs. 2009;65(5):1084-94. doi: 10.1080/ac.67.1.2146563

15. Giollo Júnior LT, Martin JFV. Índice tornozelo-braquial no diagnóstico da doença aterosclerótica carotídea. Rev Bras Hipertens. 2010;17(2):117-8.

16. Resnick HE, Lindsay RS, McDermott MM, Devereux RB, Jones $\mathrm{KL}$, Fabsitz RR, et al. Relationship of high and low ankle brachial index to all-cause and cardiovascular disease mortality: The strong heart study. Circulation. 2004;109(6):733-9. doi: 10.1161/01.CIR.0000112642.63927.54

17. Hermes BM, Cardoso DM, Gomes TJN, dos Santos TD, Vicente MS, Pereira SN, et al. Short-term inspiratory muscle training potentiates the benefits of aerobic and resistance training in 
patients undergoing CABG in phase II cardiac rehabilitation program. Rev Bras Cir Cardiovasc. 2015;30(4):474-81. doi: 10.5935/1678-9741.20150043

18. Karavidas A, Driva M, Parissis JT, Farmakis D, Mantzaraki V, Varounis C, et al. Functional electrical stimulation of peripheral muscles improves endothelial function and clinical and emotional status in heart failure patients with preserved left ventricular ejection fraction. Am Heart J. 2013;166(4):760-7. doi: 10.1016/j.ahj.2013.06.021

19. Parissis J, Karavidas A, Farmakis D, Papoutsidakis N, Matzaraki $\checkmark$, Arapi S, et al. Efficacy and safety of functional electrical stimulation of lower limb muscles in elderly patients with chronic heart failure: A pilot study. Eur J Prev Cardiol. 2015;22(7):831-6. doi: 10.1177/2047487314540546

20. Karavidas A, Parissis JT, Matzaraki V, Arapi S, Varounis C, Ikonomidis I, et al. Functional electrical stimulation is more effective in severe symptomatic heart failure patients and improves their adherence to rehabilitation programs. J Card Fail. 2010;16(3): 244-9. doi: 10.1016/j.cardfail.2009.10.023

21. Aboyans V, Criqui MH, Abraham P, Allison MA, Creager MA, Diehm C, et al. Measurement and interpretation of the
Ankle-Brachial Index: A scientific statement from the American Heart Association. Circulation. 2012,126:2890-909. doi: 10.1161/ CIR.0b013e318276fbcb

22. Sasaki M, Mitsutake Y, Ueno T, Fukami A, Sasaki KI, Yokoyama $S$, et al. Low ankle brachial index predicts poor outcomes including target lesion revascularization during the long-term follow up after drug-eluting stent implantation for coronary artery disease. J Cardiol. 2020;75(3):250-4. doi: 10.1016/j. jjcc.2019.07.015

23. Medeiros AHO, Chalegre ST, Carvalho CC. Eletroestimulação muscular: alternativa de tratamento coadjuvante para pacientes com doença arterial obstrutiva periférica. J Vasc Bras. 2007;6(2):152-62.

24. Hellsten $Y$, Nyberg M. Cardiovascular adaptations to exercise training. Compr Physiol. 2015;15;6(1):1-32. doi: 10.1002/cphy. c140080

25. Pačarić S, Turk T, Erić I, Orkić Z, Erić AP, Milostić-Srb A, et al. Assessment of the quality of life in patients before and after coronary artery bypass grafting (CABG): A Prospective Study. Int J Environ Res Public Health. 2020;17(4):1417. doi: 10.3390/ ijerph17041417 